\title{
Multilocus sequence analysis of phytopathogenic species of the genus Streptomyces
}

\author{
Correspondence \\ David P. Labeda \\ David.Labeda@ars.usda.gov
}

\author{
David P. Labeda \\ National Center for Agricultural Utilization Research, USDA-ARS, Peoria, IL 61604, USA
}

The genus Streptomyces represents an extremely important group of micro-organisms by virtue of their wide-spread presence and diversity in the environment where they play a major role in nutrient cycling and, more significantly, because of the general propensity of members of the genus to produce secondary metabolites of clinical and biotechnological importance. The identification and classification of members of this genus is difficult because of the great number of species with validly published names in the genus; at the time of writing, there were 576 and this number continues to grow each year. Within this large genus there are nine species that are considered to be phytopathogenic, including Streptomyces scabiei, Streptomyces acidiscabies, Streptomyces europaeiscabiei, Streptomyces luridiscabiei, Streptomyces niveiscabiei, Streptomyces puniciscabiei, Streptomyces reticuliscabiei, Streptomyces stelliscabiei, Streptomyces turgidiscabies (known to be the aetiological agents of scab disease in potatoes) and Streptomyces ipomoeae (causing soft rot disease in sweet potatoes). These taxa are distributed among seven different phylogenetic clades in analyses based on 16S rRNA gene sequences but the high sequence similarity in this gene among all species in the genus Streptomyces results in questions regarding the limits of its use in taxonomic resolution at the species level. Determination of DNA relatedness has served as the 'gold standard' for the definition

Two supplementary tables are available with the online version of this paper. of bacterial species (Wayne et al., 1987) but recent studies (Antony-Babu et al., 2008; Guo et al., 2008; Rong et al., 2009; Rong \& Huang, 2010) have shown that the sequencing and phylogenetic analyses of multiple housekeeping genes provides improved resolution of species-level relatedness among species of the genus Streptomyces, the results of which are in agreement with those determined from DNA-DNA hybridization studies and thus can serve as an acceptable method for discrimination of species. The present study was initiated to evaluate the relationships between phytopathogenic species of the genus Streptomyces and other closely related species using multilocus sequencing analyses.

The strains for this study were selected from the ARS (NRRL) Actinobacterial Culture Collection, where they are maintained as lyophilized preparations, and included the type strains of phytopathogenic species of the genus Streptomyces, six strains of Streptomyces isolated from either potatoes or peanuts in a previous study (Bukhalid et al., 2002) and known to contain the necl pathogenicity island conferring the ability to produce scab disease in potatoes, and 17 additional type strains observed to be phylogenetically related to the phytopathogenic species based on an unpublished study of the 16S rRNA gene sequence phylogeny of the family Streptomycetaceae.

Genomic DNA was isolated from all strains using UltraClean microbial DNA isolation kits (MoBio Laboratories) following the instructions of the manufacturer. Partial sequences 
of the housekeeping genes $a t p D, \operatorname{rec} A, r p o B$ and $\operatorname{trp} B$ were determined following the methods of Guo et al. (2008). It had been observed that two copies of the gyrB operon (one degenerate) appeared to be present in the genome sequence of Streptomyces scabiei RL87.22 available on the BacMap (Stothard et al., 2005) website (http://wishart.biology. ualberta.ca/BacMap/) at the time this study was initiated and the quality of the alignments of the sequence data for this gene from the previous studies of Guo et al.(2008), Rong et al. (2009) and Rong \& Huang (2010) appeared as if more than a single locus had been amplified and sequenced. It was decided, therefore, that this gene locus would be omitted from the present study and only those genes certain to be present as single copies would be analysed. The gene sequences of these gene loci had been determined in several published studies (Guo et al. 2008; Rong et al., 2009; Rong \& Huang, 2010). The sequences of 36 strains were obtained from the public databases to be included in the present analysis. The sequences were aligned and edited using BioEdit (Hall, 1999) and the unique allele sequences for each gene locus, including those from the published sequences and those determined in the present study, were loaded into a local implementation of the Streptomyces MLST database (http://pubmlst.org/streptomyces/) utilizing the mlstdbNET application of Jolley et al. (2004). The sequence data were exported as single gene alignments or a concatenated fourgene alignment for subsequent analysis using the TamuraNei distance model (Tamura \& Nei, 1993), using the neighbour-joining (Saitou \& Nei, 1987) and maximumparsimony (Felsenstein, 1993) algorithms with 1000 bootstrap replications (Felsenstein, 1985) in version 4 of the MEGA software package (Tamura et al., 2007), as well as the maximum-likelihood algorithm (Felsenstein, 1993) in the BioEdit program.

Phyologenetic relationships of the phytopathogenic species of the genus Streptomyces and neighbouring taxa based on $16 \mathrm{~S}$ rRNA gene sequences can be seen in Fig. 1a and b. Because of the highly conserved nature of this gene within the genus Streptomyces, there is some question regarding the limitations it has for resolution and use in species discrimination. It is of note that the majority of the phytopathogenic species are observed within the so-called Streptomyces diastatochromogenes $16 \mathrm{~S}$ rRNA gene phylogenetic cluster, or very near to it, as can be seen in Fig. 1a; this is in agreement with the traditional grouping of potato scab-producing strains by plant pathologists. Streptomyces acidiscabies, Streptomyces niveiscabiei, and Streptomyces puniciscabiei are phylogenetically distant from the $S$. diastatochromogenes cluster, as can be seen in Fig. 1b. Streptomyces luridiscabiei has recently been proposed as a later synonym of Streptomyces microflavus (Rong \& Huang, 2010) based on a multilocus sequence study and therefore its distant phylogenetic position with the Streptomyces microflavus cluster in the large Streptomyces tree is not shown.

Phylogenetic relationships of the individual housekeeping genes for this set of strains (Figs 2-5) and the tree constructed from the concatenated sequence alignment of (a)

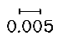

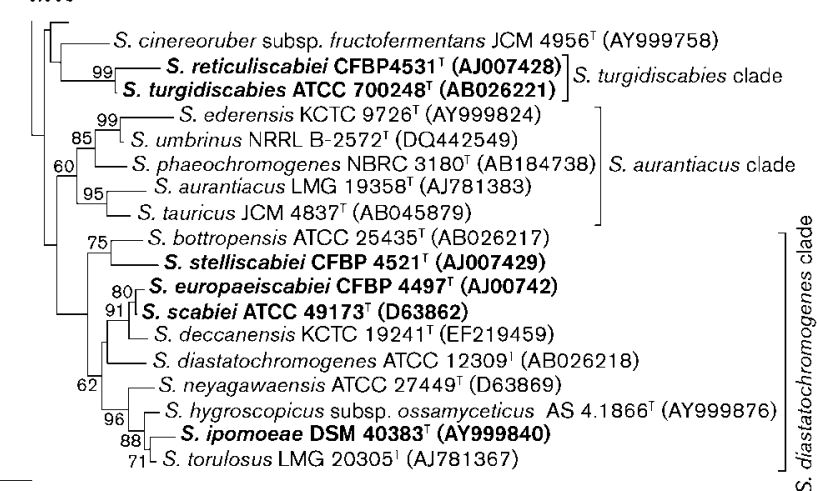

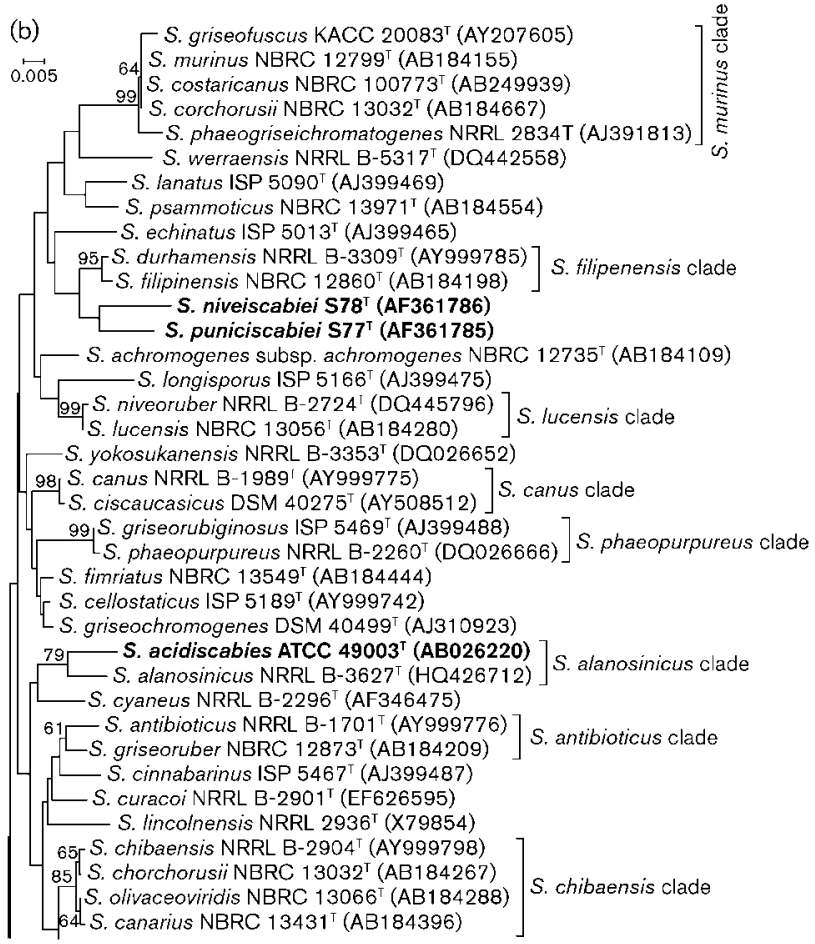

Fig. 1. Subtrees from a phylogenetic analysis calculated from sequences of the $16 \mathrm{~S}$ rRNA gene (1273 bp) showing the Streptomyces diastatochromogenes clade and neighbouring clades (a) and clades containing other phytopathogenic species and their near neighbours (b). The tree was reconstructed using the Tamura-Nei evolutionary distance method and the neighbourjoining algorithm. Known phytopathogenic species are shown in bold. Bootstrap values $>60 \%$ (based on 1000 resampled datasets) are given at nodes. Bar, 0.005 substitutions per nucleotide position.

four housekeeping genes (2111 bp) (Fig. 6) largely agreed with those inferred from analysis of $16 \mathrm{~S}$ rRNA gene sequences (Fig. 1a, 1b), but bootstrap support for these groupings was significantly higher. The topology of the phylogenetic trees constructed using the maximum-parsimony and 


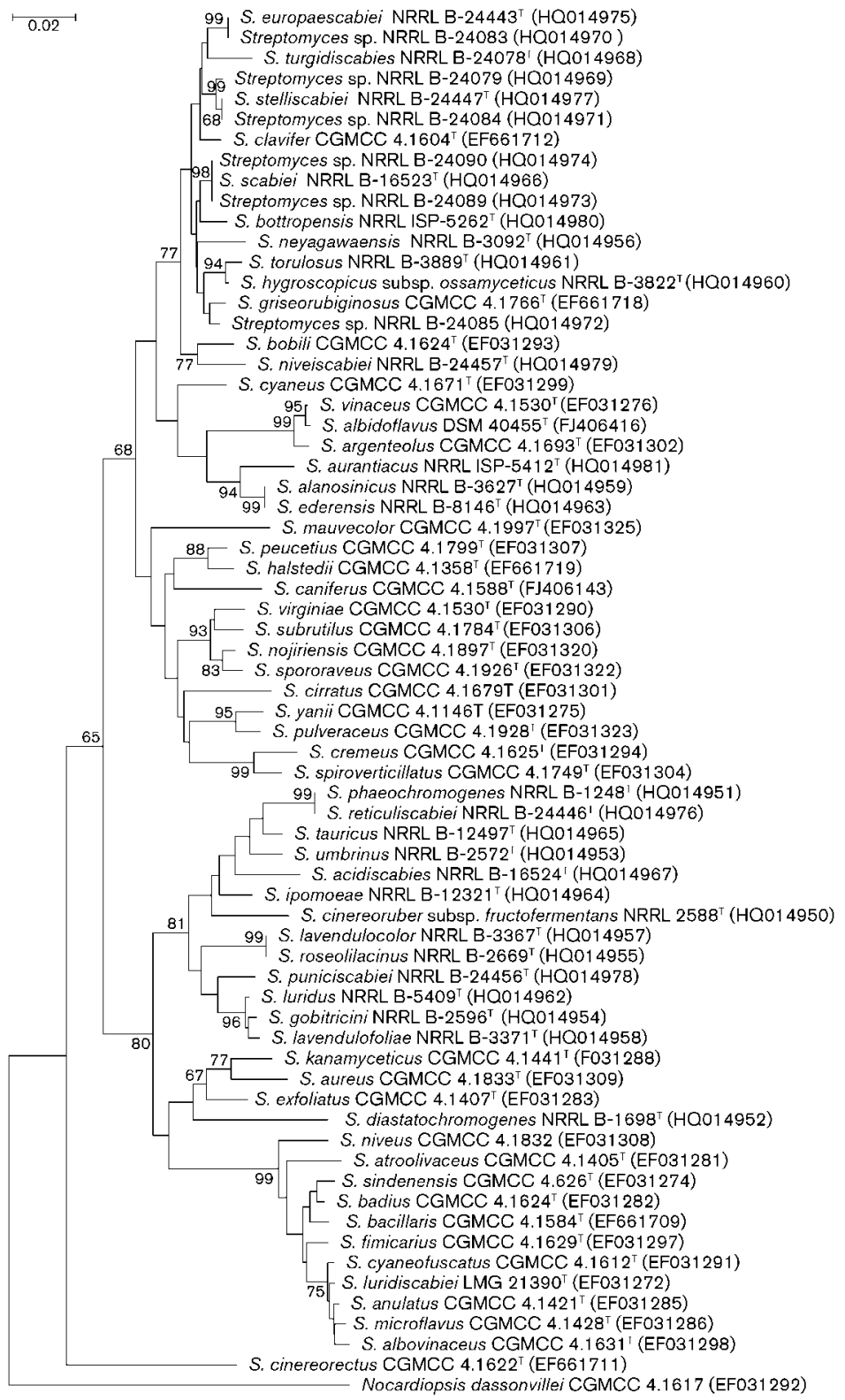

Fig. 2. Phylogenetic tree calculated from partial sequences of the atp $D$ gene (496 bp) using the Tamura-Nei evolutionary distance method and the neighbour-joining algorithm. N Bootstrap values $>60 \%$ (based on 1000 resampled datasets) are given at nodes. Bar, 0.02 substitutions per nucleotide position.

\author{
0.02 \\ Streptomyces sp. NRRL B-24089 (HO015005) \\ 91 Streptomyces sp. NRRL B-24090 (HO015006) \\ 1 S. scabiei NRRL B-16523! (HQ014998) \\ $85-$ S. bottropensis NRRL ISP-5262 1 (HO015012) \\ Streptomyces sp. NRRL B-24083 (HO015002) \\ S. europaescabiei NRRL B-24443' (HOO15007)
Streptomyces sp. NRRL B-24083(HO015002) \\ S. ipomoeae NRRL B-12321 (HQ014996) \\ S. hygroscopicus subsp. ossamyceticus NRRL B-3822 ${ }^{\top}(\mathrm{HO014992})$ \\ 99 - S. neyagawaensis NRRL B-3092' (HO014988) \\ S. torulosus NRRL B-3889' (HO014993) \\ S. alanosimicus NRRL B-3627' (HO014991) \\ S. cyaneus CGMCC 4.1671' (EF055041)
$60-$ S. clavifer CGMCC 4.1604 (EF661754) \\ Streptomyces sp. NRRL B-24085 (HQ015004) \\ 79 S. griseorubiginosis CGMCC $4.1766^{\mathrm{T}}$ (EF661760) \\ ${ }_{93}-$ S. acidiscabies NRRL B-16524 (HO014999)

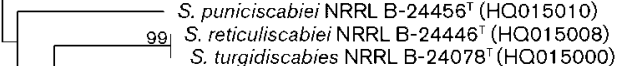

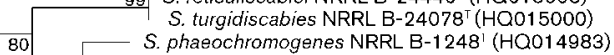 \\ $94 \quad$ S. S. ederensis NRRL B-8146 ${ }^{\top}(\mathrm{HO014995)}$ \\ 5 cinereoruber subsp. fructofermentans NRRL $2588^{\top}$ (HO014982) \\ S. aurantiacus NRRL ISP-5412' (HQ015013) \\ $90-S$. tauricus NRRL B-1 2497 (HQ014997) \\ S. diastatochromogenes NRRL B-1698 (HQ014984) \\ 88 S. vinaceus CGMCC 4.1530' (EFO55018) \\ S. albidoflavus DSM $40455^{\prime}(\mathrm{FINO} 06438)$
S. argentealus CGMCC $4.1693^{\top}$ (EFO55044) \\ S. argenteolus CGMCC $4.1693^{\top}$ (EFO55) \\ S. extoliatus Coseofiacinus NRRL B-2669T (HO014987) \\ $04 \quad$ S. S. \\ ${ }^{78} \square-$ S. lavendulocolor NRRL B-3367' (HO014989) \\ 99- S. luridus NRRL B-5409' (HO014994)

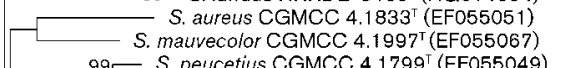 \\ $99-$ S. peucetius CGMCC 4.1799 ${ }^{\top}$ (EFO55049)
S. halstedii CGMCC $4.1358^{\top}$ (EF661761) \\ 86 S. kanamyceticus CGMCC $4.1441^{\top}$ (EFO55030)

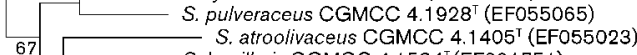 \\ 76 S. sindenensis CGMCC 4.626' (EFO312743) \\ $98 \square$ S. Badius CGMCC $4.1624^{\top}$ (EF555024)
S. albovinaceus CGMCC $4.1631^{\top}$ (EFO55040) \\ 60 S. anulatus CGMCC 4.1421 (EF (EF5027)

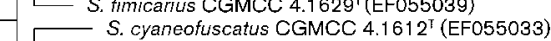 \\ 64. S. Iuridiscabiei LMG 21390 (EF055016) \\ 99 \\ S. spiroverticillatus CGMCC $4.1749^{\top}$ (EF055046)

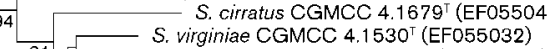 \\ S. subrutilus CGMCC $4.1784^{\top}$ (EF055048) \\ S. noijriensis CGMCC 4.18977 (EF055062) \\ 73 S. spororaveus CGMCC 4.1926
S. niveus CGMCC $4.1832($ EFO550550) \\ S. cinereorectus CGMCC 4.1622 (EF66
}

Fig. 3. Phylogenetic tree calculated from partial sequences of the recA gene (504 bp) using the Tamura-Nei evolutionary distance method and the neighbour-joining algorithm. Bootstrap values $>60 \%$ (based on 1000 resampled datasets) are given at nodes. Bar, 0.02 substitutions per nucleotide position. 
maximum-likelihood algorithms was quite similar. It was noted, however, that $S$. diastatochromogenes NRRL B-1698 was phylogenetically distant from the scab-producing taxa in all of the single gene trees as well as in the concatenated four-gene tree, while the plant pathogenic species were distributed into eight distinct clades in the latter tree (see Fig. 6). Streptomyces bottropensis NRRL ISP $-5262^{\mathrm{T}}, S$. europaeiscabiei NRRL B-24443 ${ }^{\mathrm{T}}$, S. scabiei NRRL B-16523 ${ }^{\mathrm{T}}$ and S. stelliscabiei NRRL B- $24447^{\mathrm{T}}$ were members of a statistically well-supported clade in the phylogenetic trees calculated from recA gene sequences (Fig. 3) and $r p o B$ gene sequences (Fig. 4) and were part of a larger cluster that includes clades 1, 2 and 3 in the tree (Fig. 6) calculated from the four-gene concatenated alignment. All of the species, however, could be differentiated from each other based on their concatenated four-gene phylogeny (Fig. 6) and the differences observed in their individual housekeeping gene alleles (Supplementary Table S2).

Bouchek-Mechiche et al. (2006) proposed that S. turgidiscabies and $S$. reticuliscabiei belong to the same genomic species but suggested that the names of these species be conserved because the type of scab disease produced on potatoes by each is morphologically distinct and combining the names would have caused confusion within the plant pathology community. In the present study it was observed that S. turgidiscabies and S. reticuliscabiei formed a two-membered clade distinct from the S. scabiei cluster in the four-gene tree (clade 6, Fig. 6), but they could also be clearly differentiated from one another based on differences in their $\operatorname{atp} D$ and $\operatorname{trp} B$ gene sequences (Figs 2 and 5). This was reflected in the fact that they shared the same alleles for both the recA and $r p o B$ genes but contained different alleles for the $\operatorname{atpD}$ and $\operatorname{trp} B$ genes. This observation supported the proposal of Bouchek-Mechiche et al. (2006) to maintain them as separate species.

The other phytopathogenic species were also phylogenetically distant from the S. scabiei cluster in the concatenated four-gene phylogenetic tree, with $S$. acidiscabies NRRL B$16524^{\mathrm{T}}$ and S. niveiscabiei NRRL B-24457 ${ }^{\mathrm{T}}$ forming one well-supported clade (clade 5, Fig. 6) and S. ipomoeae NRRL B-12321 $1^{\mathrm{T}}$ and S. puniciscabiei NRRL B- $24456^{\mathrm{T}}$ forming another (clade 7, Fig. 6). The members of each of these clades, however, appear to represent distinct species since the members of each clade do not share common alleles for any of the individual housekeeping genes. The housekeeping gene sequences of S. luridiscabiei NRRL B- $24555^{\mathrm{T}}$ were used as a positive control for comparison against those reported by Rong \& Huang (2010); not surprisingly, they were found to agree since they represent the same strain. In their recently published study Rong \& Huang (2010) proposed that $S$. luridiscabiei LMG $21390^{\mathrm{T}}$ represents a later synonym of $S$. microflavus, but results from the present study indicate that they are likely to represent related but distinct species because S. luridiscabiei LMG $21390^{\mathrm{T}}$ (=NRRL B-24555 ${ }^{\mathrm{T}}$ ) and S. microflavus CGMCC $4.1428^{\mathrm{T}}$ share no alleles in common for any of the four housekeeping genes.
From comparisons using the EzTaxon website (Chun et al., 2007) Streptomyces strains NRRL B-24083, NRRL B-24084, NRRL B-24085, NRRL B-24089 and NRRL B-24090 exhibited 16S rRNA gene sequence similarity values $>99 \%$ as well as DNA relatedness values of $49,50,36$, 96 and $98 \%$, respectively, with respect to S. scabiei ATCC $49173^{\mathrm{T}}\left(=\mathrm{NRRL}\right.$ B-16523 ${ }^{\mathrm{T}}$ ) as determined previously in the study of Bukhalid et al. (2002). Strain NRRL B-24079 exhibited $99.7 \% 16 \mathrm{~S}$ rRNA gene sequence similarity to the type strain of S. scabiei but genomic DNA relatedness was not determined. Analysis of the phylogenetic position of these strains based on the concatenated four-gene alignment (Fig. 6) and the specific alleles present for each housekeeping gene made it possible to identify them as members of some of the recognized phytopathogenic species. Strains NRRL B-24089 and NRRL B-24090 were identified as members of the species $S$. scabiei, as seen in Fig. 6, clade 2, having identical alleles for the atpD, recA and $r p o B$ genes. Similarly, strain NRRL B-24084 was identified as a member of S. stelliscabiei (Fig. 6, clade 1) containing identical alleles for all of the housekeeping genes. Strain NRRL B-24079, which was phylogenetically the most distant member of clade 1 , shared only the allele for the $r p o B$ gene with the other two strains and therefore may represent a new species. Strain NRRL B-24083 was identified as a member of the species $S$. europaescabiei (Fig. 6 , clade 3), the alleles for the $a t p D, r p o B$ and $\operatorname{trp} B$ genes being identical to those of the type strain, NRRL B-24443 ${ }^{\mathrm{T}}$. The identity of NRRL B-24085 was unclear at the time of writing, therefore, phylogenetic analyses of the concatenated four-gene alignment of all Streptomyces type strains may be required in order to determine if this strain belongs to another species with a validly published name.

Ritacco \& Eveleigh (2008) suggested that Streptomyces ederensis Wallhäuser et al. 1966 represents a later synonym of Streptomyces phaeochromogenes based on 16S rRNA gene sequence and partial rpoB gene sequence comparisons. However, the results of phylogenetic analyses of the concatenated four-gene dataset from the present study (see Fig. 6) and the observation that S. phaeochromogenes NRRL B-1248 ${ }^{\mathrm{T}}$ and S. ederensis NRRL B- $8146^{\mathrm{T}}$ did not contain identical alleles for any of the housekeeping genes sequenced support the continued recognition of these species as distinct. The 15 other species included in this study were also determined to be distinct based on the concatenated four-gene phylogenetic tree (Fig. 6) and novel allele contents of the housekeeping genes sequenced in the present study.

The published studies of Guo et al. (2008), Rong et al. (2009) and Rong \& Huang (2010) demonstrated the value of multilocus sequencing and phylogenetic analyses for the taxonomy and identification of members of the genus Streptomyces. This study extended their work to phytopathogenic species, and those that are phylogenetically related to these species based on 16S rRNA gene sequences, within the genus Streptomyces and confirmed 


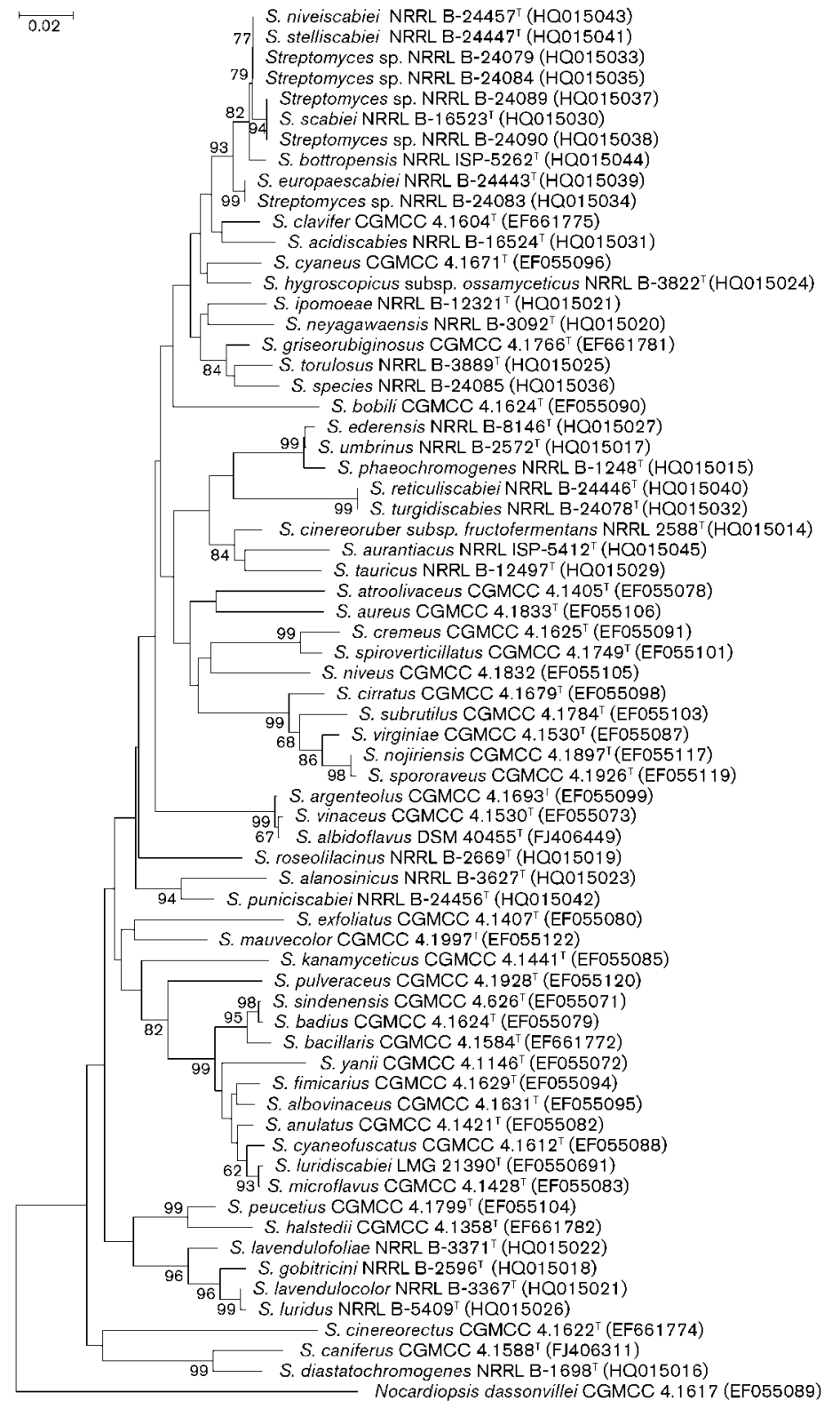

Fig. 4. Phylogenetic tree calculated from partial sequences of the $r p o B$ gene (540 bp) using the Tamura-Nei evolutionary distance method and the neighbour-joining algorithm. 0.02 substitutions per nucleotide position.

Fig. 5. Phylogenetic tree calculated from partial sequences of the $\operatorname{trp} B$ gene (571 bp) using the Tamura-Nei evolutionary distance method and the neighbour-joining algorithm. Bootstrap values $>60 \%$ (based on 1000 resampled datasets) are given at nodes. Bar, 0.02 substitutions per nucleotide position. 


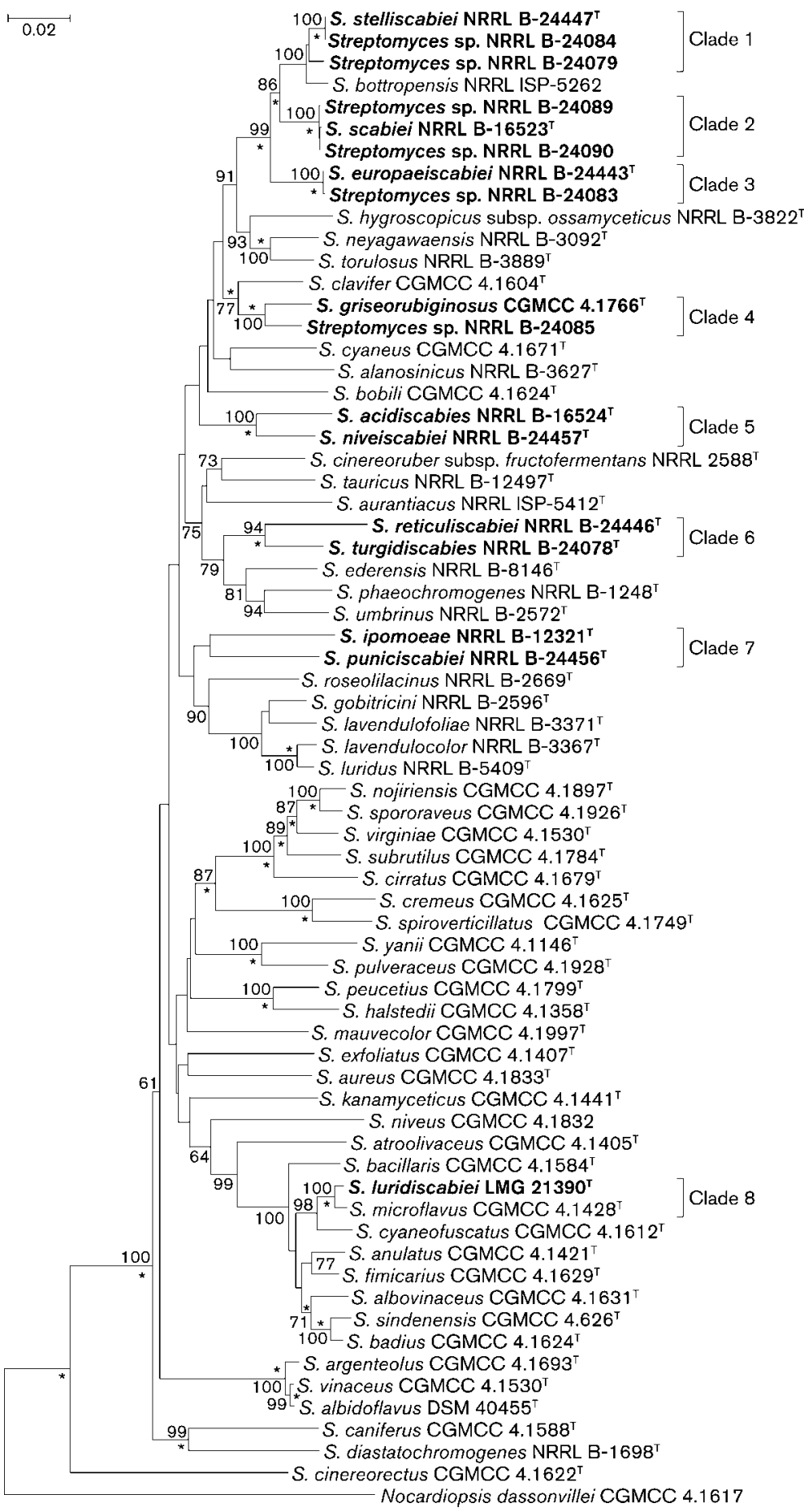

Fig. 6. Phylogenetic tree calculated from the alignment of concatenated partial sequences of the four housekeeping genes atp $D, \operatorname{rec} A, r p o B$ and $\operatorname{trp} B$ (2111 bp total) using the Tamura-Nei evolutionary distance method and the neighbour-joining algorithm. Bootstrap values $>60 \%$ (based on 1000 resampled datasets) are given at nodes. Phytopathogenic taxa are shown in bold. Asterisks indicate corresponding branches that were also recovered in the maximumlikelihood and maximum-parsimony trees. Bar, 0.02 substitutions per nucleotide position. that these species represent multiple distinct taxa. The study also demonstrated the utility of multilocus sequence analysis for the rapid and accurate identification of unknown strains isolated from diseased plant material, without the need to perform DNA-DNA hybridization experiments for definitive confirmation of identity. The data in this study appear to suggest that the four gene loci sequenced and analysed represent a minimum set of gene sequences that can prove useful for the identification of Streptomyces isolates. In the present study, identical alleles in at least three of the four housekeeping genes appears to strongly support identity at the species-level; however, this does not discount the fact that sequencing fewer genes in unknown strains might also yield useful results.

\section{Acknowledgements}

The able technical assistance of E. N. Hoekstra in the isolation of genomic DNA and determination of the sequences of the various housekeeping genes is gratefully acknowledged. Names are necessary 
to report factually on available data, however, the USDA neither guarantees nor warrants the standard of the product, and the use of the name by USDA implies no approval of the product to the exclusion of others that may also be suitable.

\section{References}

Antony-Babu, S., Stach, J. E. \& Goodfellow, M. (2008). Genetic and phenotypic evidence for Streptomyces griseus ecovars isolated from a beach and dune sand system. Antonie van Leeuwenhoek 94, 63-74.

Bouchek-Mechiche, K., Gardan, L., Andrivon, D. \& Normand, P. (2006). Streptomyces turgidiscabies and Streptomyces reticuliscabiei: one genomic species, two pathogenic groups. Int J Syst Evol Microbiol 56, 2771-2776.

Bukhalid, R. A., Takeuchi, T., Labeda, D. \& Loria, R. (2002). Horizontal transfer of the plant virulence gene, necl, and flanking sequences among genetically distinct Streptomyces strains in the Diastatochromogenes cluster. Appl Environ Microbiol 68, 738-744.

Chun, J., Lee, J.-H., Jung, Y., Kim, M., Kim, S., Kim, B. K. \& Lim, Y. W. (2007). EzTaxon: a web-based tool for the identification of prokaryotes based on $16 \mathrm{~S}$ ribosomal RNA gene sequences. Int J Syst Evol Microbiol 57, 2259-2261.

Felsenstein, J. (1985). Confidence limits on phylogenies: An approach using the bootstrap. Evolution 39, 783-791.

Felsenstein, J. (1993). Phylogeny Inference Package (PHYLIP). Version 3.5. Seattle: University of Washington.

Guo, Y., Zheng, W., Rong, X. \& Huang, Y. (2008). A multilocus phylogeny of the Streptomyces griseus $16 \mathrm{~S}$ rRNA gene clade: use of multilocus sequence analysis for streptomycete systematics. Int J Syst Evol Microbiol 58, 149-159.

Hall, T. A. (1999). BioEdit, a user-friendly biological sequence alignment editor and analysis program for Windows 95/98/NT. Nucleic Acids Symp Ser 41, 95-98.
Jolley, K. A., Chan, M.-S. \& Maiden, M. C. J. (2004). mlstdbNet distributed multi-locus sequence typing (MLST) databases. $B M C$ Bioinformatics 5, 86.

Ritacco, F. V. \& Eveleigh, D. E. (2008). Molecular and phenotypic comparison of phaeochromycin-producing strains of Streptomyces phaeochromogenes and Streptomyces ederensis. J Ind Microbiol Biotechnol 35, 931-945.

Rong, X. \& Huang, Y. (2010). Taxonomic evaluation of the Streptomyces griseus clade using multilocus sequence analysis and DNA-DNA hybridization, with proposal to combine 29 species and three subspecies as 11 genomic species. Int J Syst Evol Microbiol 60, 696-703.

Rong, X., Guo, Y. \& Huang, Y. (2009). Proposal to reclassify the Streptomyces albidoflavus clade on the basis of multilocus sequence analysis and DNA-DNA hybridization, and taxonomic elucidation of Streptomyces griseus subsp. solvifaciens. Syst Appl Microbiol 32, 314322.

Saitou, N. \& Nei, M. (1987). The neighbor-joining method: a new method for reconstructing phylogenetic trees. Mol Biol Evol 4, 406425.

Stothard, P., Van Domselaar, G., Shrivastava, S., Guo, A., O'Neill, B., Cruz, J., Ellison, M. \& Wishart, D. S. (2005). BacMap: an interactive picture atlas of annotated bacterial genomes. Nucleic Acids Res 33 (Database issue), D317-D320.

Tamura, K. \& Nei, M. (1993). Estimation of the number of nucleotide substitutions in the control region of mitochondrial DNA in humans and chimpanzees. Mol Biol Evol 10, 512-526.

Tamura, K., Dudley, J., Nei, M. \& Kumar, S. (2007). MEGA4: molecular evolutionary genetics analysis (MEGA) software version 4.0. Mol Biol Evol 24, 1596-1599.

Wayne, L. G., Brenner, D. J., Colwell, R. R., Grimont, P. A. D., Kandler, O., Krichevsky, M. I., Moore, L. H., Moore, W. E. C., Murray, R. G. E. \& other authors (1987). International Committee on Systematic Bacteriology. Report of the ad hoc committee on reconciliation of approaches to bacterial systematics. Int J Syst Bacteriol 37, 463-464. 